\title{
MORFOLOGIA Y BIOLOGIA DE BostryX conspersus (SOWERBY) (MOLLUSCA, BULIMULIDAE) EN LAS LOMAS COSTERAS DEL PERU CENTRAL
}

\author{
Rina Ramírez ${ }^{1}$
}

\begin{abstract}
Morphological and biological observations on populations of the land snail Bostryx conspersus, in three "Lomas" of the Central Coast of Peru, were carried out between 1979 and 1982. Three shell color varieties of $\mathbf{B}$. conspersus are reported herein, as well as its aestivation, activity and reproduction strategies. The possible interactions of $\mathbf{B}$. conspersus with a dipteron (Scaptomyza sp.; Drosophilidae) and a nematode species are also discussed.
\end{abstract}

\section{INTRODUCCION}

En el desierto costero del Perú y norte de Chile se desarrollan ecosistemas peculiares denominados "Lomas" (Ono, 1982; Oka \& Ogawa, 1984), bajo la influencia de la humedad traída por las neblinas advectivas de la costa. Ellenberg (citado por Oka \& Ogawa, 1984) las denominó "oasis de neblina". Las "Lomas" se caracterizan por su estacionalidad muy marcada, con una "Epoca seca" (diciembre-abril) de baja humedad atmosférica (52-98\%) y del suelo (1.57-3.10\%), y máximas temperaturas del aire $\left(19-33^{\circ} \mathrm{C}\right)$ y del suelo $\left(23-55^{\circ} \mathrm{C}\right)$, y otra totalmente opuesta, la "época húmeda" (julio-setiembre), con máximos valores de humedad (85-100\% -aire; $12-15 \%$ - suelo) y mínimos de temperatura $\left(13-16^{\circ} \mathrm{C}\right.$ 2ire; $15-184$

aire; $15-18^{\circ} \mathrm{C}$-suelo), favoreciendo el desarrollo de la vegetación herbácea y supraterrestre (Torres \& López, 1981). Los gasterópodos se han adaptado a estas condiciones cambiantes, desarrollando diversas estrategias para sobrevivir a la "época seca" (Pulido \& Ramírez, 1982). En tales ecosistemas, la familia Bulimulidae presenta la mayor diversidad de especies (Aguilar, 1979, 1985), siendo Bostryx conspersus (Sowerby, 1833) la especie más común en las Lomas del centro del Perú.

En el presente trabajo se estudió la morfología de la conchilla, los órganos paliales, la genitalia y el huevo de Bostryx conspersus, así como sus estrategias de actividad, estivación y reproducción, y su interacción con dos especies de invertebrados (un díptero y un nemátodo). 


\section{MATERIAL Y METODOS}

Se realizaron salidas mensuales, de dos días promedio en duración, a las Lomas de Lachay (en 1979-1980), Iguanil (1982), y Pacta (1982), todas en el departamento de Lima, Perú (Torres \& López, 1981). El muestreo fue cualitativo y randomizado (Paul, 1975), y la búsqueda por observación visual (Southwood, 1978), desde la 0700 hasta las 1800 (hora local). Los caracoles fueron muertos por ahogamiento en agua de caño, en 24 horas. Las disecciones se hicieron bajo agua y los dibujos con cámara lúcida. Para la disección del huevo, se utilizó una lámina excavada con agua, presionándolo gradualmente por medio de una laminilla soportada con un estilete por uno de sus lados, hasta que la cáscara se rompiera y saliera intacto el resto del huevo. Los huevos se mantuvieron bajo condiciones ambientales, enterrados en suelo humedecido perpermanetemente, en placas Petri.

En los cálculos numéricos se da la media $(\bar{x})$ de la muestra $(\mathrm{n})$ con 0.95 límite de confianza (s), y los rangos (máx, mín).

\section{RESULTADOS}

\section{Morfologia}

a) Conchilla (fig. 3a-c)

En las tres poblaciones estudiadas (Lomas de Lachay, Iguanil y Pacta) han sido halladas tres variedades respecto a la coloración: (1) conchilla de color castaño claro con manchas redondeadas blancas, que en conjunto le dan una coloración blanquecina (Fig. 3b); (2) castaño intenso con manchas puntiformes a lineales, muy finas (Fig. 3a); y (3) castaño uniforme (Fig. 3c). La primera es la más común y las otras dos fueron escasas. En los individuos recién eclosionados no se notó esta variación, siendo todos castaño claros a amarillo opacos.

\section{b) Organos paliales ( fig.1)}

El pericardio mide aproximadamente $1 / 7$ del largo del nefridio, que tiene forma de triángulo isósceles. La vena pulmonar principal es prominente y ramificada en su extremo anterior; las venaslaterales están bien desarrolladas y el manto en esta región muestra una apariencia negruzca. El uréter primario o adrenal es prominente en su región proximal, adelgazándose hacia la distal; el ureter secundario o adrectal es cerrado.

\section{c) Genitalia (Figs. 2 y 7 )}

El ovotestis está formado por lóbulos a manera de racimos, que se unen en el conducto hermafrodítico, de cuyo extremo distal sale el talón o cámara de fertilización; ésta es muy notoria, larga y plegada sobre la parte media cóncava de la glândula de la albúmina. La glândula es de coloración variable, yendo de amarillo cremoso a anaranjado intenso y marrón rojizo, siendo 

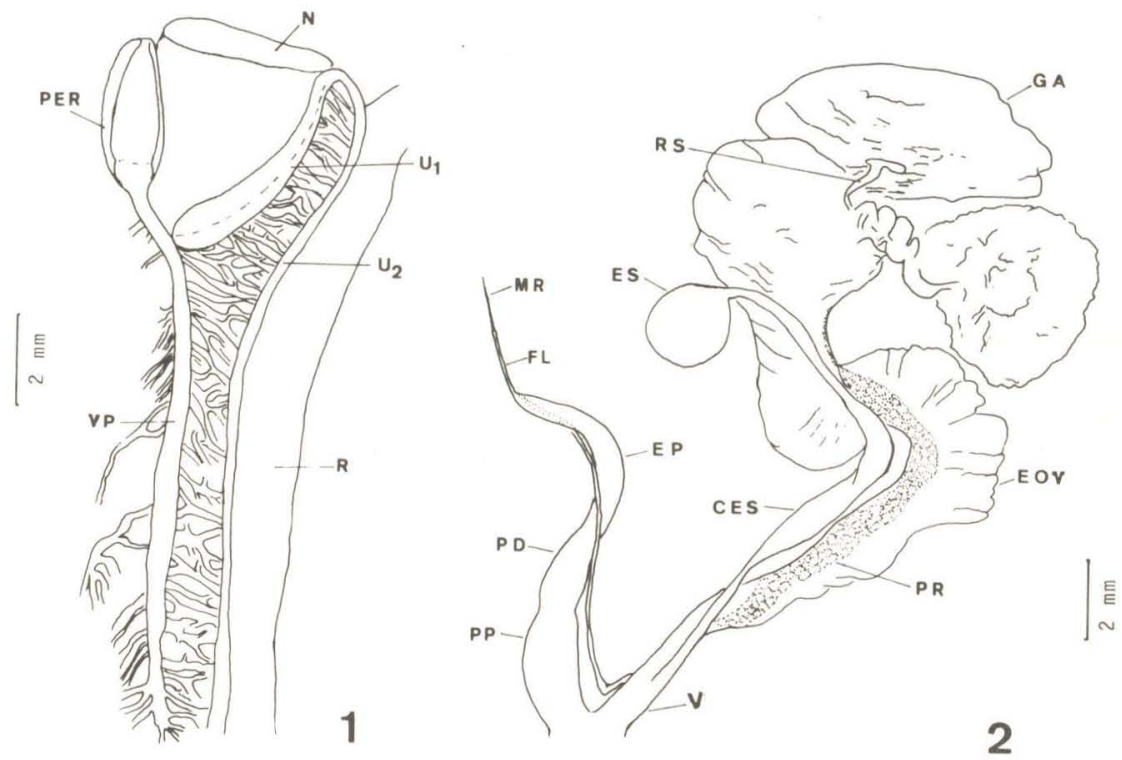

FIGS. $1-2-1$. Organos paliales y 2. Genitalia de $B$. conspersus. CES: conducto de la espermateca; EOV: espermo'viducto; EP: epiphallus; ES: espermateca; FL: flagelo; $\mathrm{GA}$ : glándula de la albúmina; MR: músculo retractor; N : nefridio; PD:porción distal del pene; PER: pericardio; PP: porción proximal del pene; PR: próstata; R: recto; RS: receptáculo seminal; $\mathrm{U}_{1}$ : uréter primario; $\mathrm{U}_{2}$ : uréter secundario; $\mathrm{V}$ : vagina; $\mathrm{VP}$ : vena pulmonar principal. $($ Escalas $=2 \mathrm{~mm})$.

fácilmente observable a través de la conchilla; las coloraciones más intensas fueron observadas en la mayoría de los indivíduos adultos activos.

Las paredes del espermoviducto presentan pliegues perpendiculares al eje longitudinal, y la región prostática es angosta; ésta no alcanza el conducto hermafrodítico, una zona ensanchada cuyo diámetro es dos veces el del espermoviducto. El conducto de la espermateca es tan largo como el espermoviducto; los $2 / 4$ medios son algo más ensanchados que el resto, pero cuando contiene al espermatóforo, se dilata considerablemente. La espermateca es globosa. La vagina es relativamente grande, aproximadamente $1 / 3 \mathrm{del}$ oviducto.

El pene no presenta vaina, se constriñe antes de llegar al epiphallus, volviéndose a dilatar hacia la transición al epiphallus (porción distal del pene), que es poco notoria; el epiphallus es subcilíndrico, agudizándose hacia el flagelo, que es muy corto y delgado (ca. 1/10 del largo del phallus).

El espermatóforo es un tubo subcilíndrico, sin ornamentaciones, enrollado a lo largo de un eje longitudinal, ocupando los $2 / 4$ medios del conducto de la espermateca (Fig. 7). 


\section{d) Huevo (Figs. 4-6; Tabla 1)}

Los huevos son esféricos, de ca. $1.5 \mathrm{~mm}$ de diámetro; al momento de ser depositados son de color anaranjado intenso, tornándose amarillo claros luego de unos minutos. Poseen tres membranas terciarias: (1) la externa (cáscara), está impregnada de cristales (Figs. 4 y 6), es translúcida y resistente, pero flexible, siendo la que da coloración amarillenta al huevo; (2) la intermedia es muy delgada y transparente (Figs. 5 y 6), poco resistente, estando separada de la externa e interna por espacios muy reducidos, llenos de sustancia mucilaginosa; y (3) la interna es fina y transparente (Fig. 5), y la más resistente, limitando un espacio que llena casi todo el diámetro del huevo con líquido (albúmina) que contiene al embrión; tal líquido es de color anaranjado en huevos recién puestos, tornándose luego incoloro.

TABLA 1 - Tamaño de nidada (N) y tiempo de incubación (i) de huevos de Bostryx conspersus.

$\begin{array}{lcc} & \mathrm{N} & \mathbf{i} \\ \mathrm{n} 9 & & \\ \mathrm{x} & 18.67 & 19.56 \\ \mathrm{~s} & 5.72 & 1.68 \\ \mathrm{x}_{\text {máx }} & 34 & 22 \\ \mathrm{x}_{\text {mín }} & 10 & 17\end{array}$

\section{Biologia}

\section{a) Estivación}

Durante los meses de enero a mayo no se observó ningún individuo vivo de B. conspersus en la superficie. Se encontró individuos enterrados a más de $2 \mathrm{~cm}$ de profundidad, adyacentes a la base de subarbustos y rocas, muy pocas veces junto a árboles. Estos individuos presentaron epifragma blanquecino y opaco, y el pie muy retraído.

\section{b) Actividad}

Sólo de junio a diciembre fueron hallados individuos activos de $B$. conspersus. Durante este periodo se los encontró en tres estados o categorías de actividad: (1) activos, en movimiento sobre el suelo, rocas y vegetacion herbácea y arbustiva; (2) inactivos, adheridos al substrato; y (3) inactivos, semienterrados o enterrados superficialmente $(1-2 \mathrm{~cm})$, en la base de vegetación y rocas. Estos individuos presentaban un epifragma muy débil y translúcido, y el pie muy cerca a la abertura. En el laboratorio exhibieron estos mismos patrones de 
Vol. 5(4), 1988

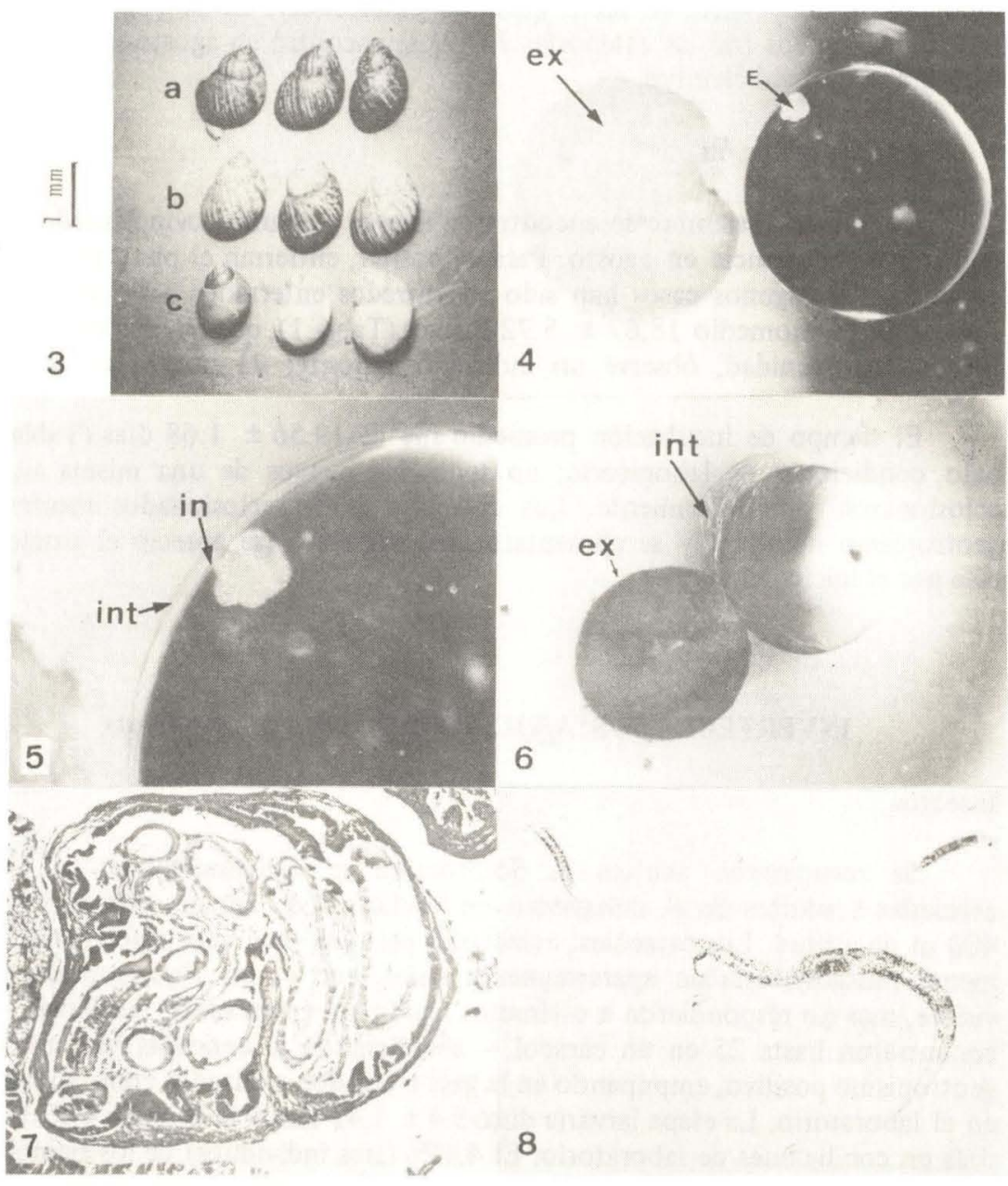

FIG. 3a-c - Variedades de Bostryx conspersus encontradas en las Lomas del Perú central; a y c son raras. $($ Escala $=1 \mathrm{~cm})$.

4 - 6 - Huevo de $B$. conspersus con embrión de nueve días. 4 y 5 : con la membrana ex terna ex traída; 6 : con las membranas ex terna e intermedia removidas. E:

embrión; ex: membrana externa; int: membrana intermedia; in: membrana interna. $4: 28 X ; 5: 45 X ; 6: 15 X$.

7 - Secciones del espermatóforo de $B$. conspersus en el conducto de la espermateca (parte media). (Hematoxilina - Eosina) 50X.

8 - Nemátodo encontrado en huevos y en individuos muertos de $B$. conspersus, y en forma libre en el suelo. 133X. 
Revta bras. Zool.

comportamiento, pero mayormente se mostraron inactivos, saliendo de tal estado luego de ser humedecidos directamente (cuando en la superficie) o haber humedecido muy bien el suelo (cuando enterrados). El mayor número de individuos activos (de las categorías 1 y 2 ) se encontró en agosto-setiembre, el menor en junio y diciembre.

\section{c) Reproducción}

De julio a noviembre se encontraron huevos y adultos ovipositando, pero con mayor frecuencia en agosto. Para ovipositar, entierran el pie y parte de la conchilla (en algunos casos han sido encontrados enterrados a ras del suelo). Depositan en promedio $18.67 \pm 5.72$ huevos (Tabla 1), que quedan enterrados. En una oportunidad, observé un individuo depositar 21 huevos en $60 \mathrm{~min}$.

El tiempo de incubación promedio fue de $19.56 \pm 1.68$ días (Tabla 1), bajo condiciones de laboratorio; no todos los huevos de una misma nidada eclosionaron simultaneamente. Los caracoles recién eclosionados mostraron geotropismo negativo, y se alimentaron del substrato (se apreció el pasaje de éste por el inicio del buche).

\section{INVERTEBRADOS ASOCIADOS A Bostryx conspersus}

\section{Insectos}

Se recuperaron adultos de Scaptomyza sp. (Drosophilidae) de larvas asociadas a adultos de $B$. conspersus en las Lomas de Iguanil, por encima de $400 \mathrm{~m}$ de altitud. Los caracoles, colectados del suelo con vegetación (principalmente musgos), estaban aparentemente sanos, con el pie algo retraído pero visible, mas no respondieron a estímulos mecánicos (punzadas). Las larvas - se encontraron hasta 25 en un caracol - abandonaron la conchilla ya vacía con geotropismo positivo, empupando en la gasa o algodón utilizados como substrato en el laboratorio. La etapa larvaria duró $5.4 \pm 1.42$ días y la de pupa $4.75 \pm 0.8$ días en condiciones de laboratorio. El $4.41 \%$ (tres individuos) de los ejemplares de $B$. conspersus registrados el 17 ix 82 contenían larvas y huevos de Scaptomyza sp.

\section{Nemátodos}

Larvas rabditiformes (Fig. 8), posiblemente del orden Rhabditoidea, fueron observadas alimentándose de la albúmina de huevos con cáscara rota, así como horadando la cáscara de huevos sanos. También se las encontró alimentándose de la parte blanda de individuos muertos, recién eclosionados y adultos, así como en forma libre en el suelo. 


\section{DISCUSION}

La conchilla de Bostryx conspersus carece de coloración llamativa, variando de blanquecina a castaña, distinguiéndose en los adultos tres variedades. No se apreciaron estas variedades en individuos recién eclosionados, cuya coloración (castaño claro a amarillo opaco) es dada por el periostraco (Owen, 1965; Matos, 1981, 1984). La variación en coloración ha sido apreciada también en otras poblaciones, como mencionan Hidalgo (1900) (para el "Cerro de las Conchitas en las cercanías de Lima") y Weyrauch (1967a).

En un año climáticamente normal de "Lomas", B. conspersus estiva durante 5 meses (enero-mayo), coincidiendo con la "época seca", cuando la temperatura del aire y del suelo alcanzan sus máximos valores, mientras la humedad relativa y del suelo, así como la cobertura vegetal, son mínimas (Torres \& López, 1981). Estiva enterrado, lejos de la superficie (Pulido \& Ramírez, 1982) donde la temperatura es menor; en ningún caso se halló individuos vivos "en las resquebrajaduras de las rocas o en la parte baja de ellas", como cita Weyrauch (1967b), sólo conchillas vacías.

Bostryx conspersus se muestra activo durante siete meses, presentando tres categorías de actividad bien definidas, como sucede en otras especies (Nisbet, 1974; Pollard, 1975): en movimiento, inactivo en la superficie, e inactivo enterrado. No todos los individuos "despiertan" o vuelven a estivar al mismo tiempo. El período de actividad empieza en junio, junto con el inicio de la "época húmeda" de las Lomas, y termina en diciembre, al comienzo de la "época seca", encontrándose el mayor número de individuos activos en agostosetiembre. Esto coincide con el apogeo de la "época húmeda".

Aunque nunca se observó individuos de $B$. conspersus en cópula, se puede asumir, por la presencia de huevos en julio, que copulan prácticamente desde que empieza su actividad, ovipositando cuando aún las condiciones de humedad del suelo no son las mejores. Pero la mayoría oviposita en agosto y setiembre, cuando las condiciones del suelo son propicias para el desarrollo del huevo (alta humedad y baja temperatura) disminuyendo así la probabilidad de mortandad por desecación (Owen, 1965; Pollard, 1975; Ramírez, 1984). Los nuevos individuos tendrían entonces mayores probabilidades de sobrevivir y alcanzar por lo menos el estadío juvenil antes de entrar en estivación.

Entre los pulmonados terrestres, Tompa (1979) diferencia a caracoles ovíparos, caracoles que retienen sus huevos, caracoles ovovivíparos y ovovivíparos facultativos. Según tal clasificación, B. conspersus correspondería al grupo de los ovíparos por su forma de depositar los huevos (en "assemblyline fashion"), y por encontrarse entonces el cigote en sus primeras divisiones.

El drosofílido obtenido de especímenes adultos de B. conspersus, Scaptomyza sp., corresponde al tipo "saprófago", caracterizado porque la larva se alimenta y completa su desarollo en un solo caracol (Beaver, 1972, 1977). Nunca antes una especie de dicho género ha sido registrada en habitats similares (Wirth, com. pers.), aunque sí se ha registrado otros drosofílidos en varios tipos de carroña, pero tan sólo una (Drosophila tripunctata) en la de un pulmonado (babosa) (Kneidel apud Beaver, com. pers.). 
Revta bras. Zool.

Stephenson \& Knutson (1966) indican que 27 especies de nemátodos de vida libre han sido reportadas como parásitos de pulmonados (Arion spp.). El nemátodo encontrado en forma libre en el suelo, en las Lomas de Iguanil, y también asociado a huevos y caracoles, podría corresponder a un parásito facultativo de huevos e individuos recién eclosionados, y ser así uno de los principales causantes biológicos de la mortandad de $B$. conspersus en sus primeros estadíos.

\section{AGRADECIMIENTOS}

Expreso mi profundo agradecimiento a la Dra. Estela Castillo por el apoyo y sugerencias brindados para la realización del presente trabajo; asimismo, al Centro de Investigaciones de Zonas Aridas de la Universidad Nacional Agraria (CIZA - UNA) por su apoyo económico. A los Blgos. Víctor Pulido, Diana Silva y Marco Espinoza por su ayuda en el trabajo de campo. A los Drs. W. Wirth, W. Dale y L. Pilares por la identificación del díptero, y a la Dra. L. Sarmiento por la del nemátodo. Al Dr. Víctor Ishiyama por las facilidades brindadas para el trabajo histológico. Al Prof. Jaime Descailleaux y a los Srs. G. Llerena, A. Rodríguez y M. Valderrama por la preparación de las microfotografías. Al Dr. Gerardo Lamas por su paciente revisión del manuscrito.

\section{REFERENCIAS}

Aguilar, P. 1976. Fauna desértico-costera peruana. I. Invertebrados más frecuentes en las Lomas. Revta per. Ent. 19(1):67-70.

Aguilar, P. 1985. Fauna de las Lomas Costeras del Perú. Bol. Lima 7(41):17-28.

Beaver, R. 1972. Ecological studies on Diptera breeding in dead snails. I. Biology of the species found in Cepaea nemoralis (L.). Entomologist 105(1305):41-52.

Beaver, R. 1977. Non-equilibrium 'island' communities: Diptera breeding in dead snails. J. anim. Ecol. 46(3):783-798.

Hidalgo, J. 1900. Descripción de los Moluscos recogidos por la Comisión Científica enviada por el Gobierno Español a la América Meridional. Mems. R. Acad. Ci. exact., fís. nat. Madrid 19(1):1-608.

Matos, R. 1981. Interacção Génica em Caracteres Pigmentares da Concha de Helix aspersa. Portug. Acta biol. (A) 17(1/4):37-79.

Matos, R. 1984. Genetics of shell ground colour in Helix aspersa. I. Colour locus, uniform and their intereactions. Heredity 53(1):11-20.

Nisbet, R. 1974. The life of Achatinidae in London. Proc. malac. Soc. Lond.41(3):171184.

Oka, S.\& H. Ogawa. 1984. The distribution of Lomas Vegetation and its climatic environments along the Pacific coast of Peru. Geogr. Rep. Tokyo metrop. Univ. 19:113125. 
Ono, M. (Ed .) 1982. A preliminary report of Taxonomic and Ecological Studies on Lomas Vegetation in the Pacific Coast of Peru. Makino Herbarium, Tokyo Metropolitan University, 80pp.

Owen, D. 1965. A population study of an equatorial land snail, Limicolaria martensiana (Achatinidae). Proc.zool. Soc. Lond. 144:361-382.

Paul, C. 1975. The ecology of Mollusca in ancient woodland. 1. The fauna of Hayley Wood, Cambridgeshire. J. Conch. 28(5):301-327.

Pollard, E. 1975. Aspects of the ecology of Helix pomatia L. J.anim. Ecol. 44(1):305-329.

Pulido, V.\& R. Ramirez. 1982. Distribución y actividad estacional de los caracoles terrestres de las Lomas de Lachay - Lima. Bitác. biol. (Lima). 1(1):52.

Ramirez, R. 1984. Aspectos de la Ecología de Bostryx conspersus (Sowerby, 1833) (Mollusca, Bulimulidae) en las Lomas de Iguanil, Huaral-Lima. Trabajo para optar Título Profesional de Biólogo. Fac. de CC.BB., Univ. Nac. Mayor de San Marcos, 63pp.

Southwood, T. 1978. Ecological methods. Chapman and Hall, London. (Ed. 2), 524pp.

Stephenson, J. \& L. Knutson. 1966. A Résumé of Recent Studies of Invertebrates Associated with Slugs. J. econ. Ent. 59(2):356-360.

Tompa, A. 1979. Oviparity, egg retention and ovoviviparity in pulmonates. J. Moll. Stud. 45(2): 185-160.

Torres, J. \& C. Lopes. 1981. Productividad primaria en las Lomas de la Costa Central del Perú. Bol. Lima 2(14):54-63.

Weyrauch, W. 1967a. Treinta y ocho nuevos Gastrópodos terrestres de Perú. Act. zool. Lilloana 21:343-455.

Weyrauch, W. 1967b. Apuntes de Clase, Zoología Sistemática, 2da.parte. P. Aguilar, Lima (mimieo.). 